\title{
SERUM MYELOPEROXIDASE, MALONDIALDEHYDE, ALPHA-SYNUCLEIN LEVELS IN PATIENTS WITH EPILEPSY
}

\author{
Fatma Şimşek $^{1}$, Mustafa Ceylan ${ }^{1}$, Seda Aşkın² ${ }^{2}$ Ahmet Kızıltunç ${ }^{2}$ \\ Correspondence: klamaks@hotmail.com

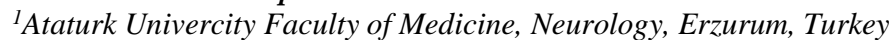 \\ ${ }^{2}$ Ataturk Univercity Faculty of Medicine, Biochemistry, Erzurum, Turkey
}

\section{Article History:}

Received: January 22, 2021

Accepted: April 21, 2021

Published: July 1, 2021

\section{Cite this as:}

Şimşek F, Ceylan M, Aşkın S,

Kızıltunç A. Serum

myeloperoxidase,

malondialdehyde, alpha-

synuclein levels in patients with

epilepsy. Malang Neurology

Journal; 2021.7:93-97.

http://dx.doi.org/10.21776/ub.mnj

.2021.007.02.2

\section{ABSTRACT}

Background: Processes such as neurodegeneration, hypoxia, blood brain barrier dysfunction and oxidative changes are effective for epileptogenesis.There is no non-invasive biomarker that can be used in the follow-up of patients with epilepsy, which is a neurodegenerative disease.

Objective: In our study, it was aimed to investigate the relationship between inflammatory, oxidative, neurodegenerative processes, and antiepileptic use in patients with epilepsy.

Methods: The groups were formed from the patients who were followed up in the epilepsy outpatient clinic between April 2019-June 2019, and the age-gender-matched control group.The study included 30 patients and 30 healthy volunteers. Venous serum samples were collected from groups to study myeloperoxidase, malondialdehyde and alpha-synuclein.

Results: The levels of myeloperoxidase and malondialdehyde were higher in the control group and this difference was statistically significant $(\mathrm{p}=0.003, \mathrm{p}<0.001)$. The level of $\alpha$-syn was higher in the epilepsy group and there was no statistically significant difference between the two groups $(\mathrm{p}=0.52)$. There was a positive correlation between the $\alpha$-syn level and disease duration and as the disease duration increased, the level of $\alpha$-syn increased ( $r=0.379, p=0.03$ ).

Conclusion: Although the $\alpha$-syn level increases with the duration of the disease in epilepsy patients, it is not a suitable parameter for use as a biomarker in the follow-up.

Keywords: Epilepsy, myeloperoxidase, malondialdehyde, alpha-synuclein

\section{Introduction}

Epileptogenesis describes structural changes leading to seizure activity in brain. In recent years, many reasons such as neurodegeneration, blood-brain barrier disorder, changes in the glutaminergic system, hypoxia, oxidative changes have been suggested to explain epileptogenesis. ${ }^{1-5}$ The mechanism in epileptogenesis is uncertain and studies are suggesting an important role of inflammation in the etiology. ${ }^{6}$ Leukocytes, which are one of the important components of inflammation, are important during epileptogenesis because it has been shown in experimental studies that depletion of neutrophils prevents seizure induction and development of epilepsy. ${ }^{7}$

Myeloperoxidase (MPO) is an inflammatory enzyme found in the granules of neutrophils. ${ }^{8}$ MPO plays a role in the pathogenesis of epilepsy and many different neurological diseases. Considering oxidative stress, tissue damage, and inflammation as cause and effect-related cycles, the idea that an important mechanism like the inflammatory system in epileptogenesis is oxidative changes can be put forward. Increased free radicals have been reported to cause neuronal damage. ${ }^{9}$

It has also been reported that the nervous system is susceptible to oxidative stress-induced damage, especially due to the polyunsaturated fatty acid content prone to lipid peroxidation. ${ }^{10}$ In several studies, lipid peroxidation has been associated with neurological and neurodegenerative diseases. ${ }^{11}$ Malondialdehyde (MDA) is a lipid peroxidation product.

Alpha-synuclein ( $\alpha$-syn) is a small, acidic protein and is localized in the synaptic terminals.12 Inflammation and oxidative stress have been suggested to trigger the phosphorylation and aggregation of $\alpha$-syn.13 It is mainly localized in the neocortex, striatum, and thalamus hippocampus, thalamus, and cerebellum. ${ }^{14}$

Epilepsy is mainly diagnosed clinically and the diagnosis is supported by electroencephalography. There is no objective biomarker used to indicate the course and progression of the disease in epilepsy. In our study, it was aimed to investigate the inflammatory, oxidative, neurodegenerative process, and their relationship with antiepileptic use in epilepsy patients.

\section{Methods}

\section{Patient selection}

The groups were formed from the patients who were followed up in the epilepsy outpatient clinic between April 2019-June 2019, and the age-gender-matched control 
group. The ILAE classification was used for the epilepsy diagnosis of the patients. A study invitation was sent to patients who had no other systemic disease with follow-up in the epilepsy clinic, who were between the ages of 18-55, who were noted to have no structural lesions in their neuroimaging (3 T MRI, coronal flair slices). Venous blood samples were received from the first 30 patients meeting the criteria and 30 healthy volunteers. The seizure types, duration of disease, seizure frequency (annual number of attacks), duration of drug use (months, patients who had used monotherapy for at least 6 months and patients who had received polytherapy for more than 6 months), and drug doses of the patients were recorded. For the study, written consent from the participants and approval from the local ethics committee was obtained.

Collection and study of serum samples: For myeloperoxidase, malondialdehyde, and alpha-synuclein levels, $5 \mathrm{ml}$ of the venous blood sample was taken from the patients and healthy volunteers and centrifuged at $4000 \mathrm{rpm}$ for 5 minutes. The serums were placed in two separate Eppendorf tubes and stored at $-80{ }^{\circ} \mathrm{C}$ until the study.

\section{Myeloperoxidase}

The assay is based on the kinetic measurement of the absorbance of the yellowish-orange complex at $460 \mathrm{~nm}$ wavelength, which results from the oxidation of myeloperoxidase (MPO) and o-dianisidine in the presence of $\mathrm{H} 2 \mathrm{O} 2 .{ }^{15}$

\section{Alpha-synuclein}

The level of $\alpha$-syn was studied using the ELISA method (SunRed cat no: 201-12-1314). The analysis results were calculated as $\mathrm{ng} / \mathrm{ml}$ according to the manufacturer's instructions.

\section{Malondialdehyde}

The measurement principle of malondialdehyde (MDA) which results from lipid peroxidation is based on the measurement of the absorbance of the pink compound formed by the reaction of MDA and thiobarbituric acid (TBA) at $532 \mathrm{~nm} .{ }^{16}$

\section{Statistical analysis}

All analyses were performed with SPSS 20. Numeric variables were expressed as mean $\pm \mathrm{SD}$, minimummaximum. Categorical variables were shown as $\mathrm{n}(\%)$. The t-test was used for comparisons. Pearson's rank correlation coefficient was calculated to determine the relationship between $\alpha$-syn levels and disease duration. The level of significance was set at $\mathrm{p}<0.05$. It is written using Times New Roman 10 with single space and each new paragraph indents in 3 pt.

\section{Results}

The group's age was $29.23( \pm 10.9)$ years. 40 of the 60 cases were female. Demographic and clinical data of epilepsy patients are shown in Table 1. There was no statistically significant difference between the male and female patients in terms of the MPO, MDA, and $\alpha$-syn levels $(p=0.257$, $\mathrm{p}=0.861, \mathrm{p}=0.290)$. The levels of MPO and MDA were higher in the control group and this difference was statistically significant (Table 2). The level of $\alpha$-syn was higher in the epilepsy group and there was no statistically significant difference between the two groups. There was no statistically significant correlation between the annual number of attacks $\alpha$-syn level ( $\mathrm{p}=0.82, \mathrm{r}=-0.04)$. There was no statistically significant difference between the monotherapy and polytherapy groups in terms of the MPO, MDA, and $\alpha$-syn levels (Table 3 ). There was a positive correlation between the $\alpha$-syn level and disease duration and as the disease duration increased, the level of $\alpha$-syn increased $(\mathrm{r}=0.379, \mathrm{p}=0.03)$. When we divided the patients into two groups as primary generalized seizure and secondary generalized seizure, there was no statistically significant difference between the seizure type and MPO, MDA, $\alpha$-syn (Table 4).

Table 1. Demographic and clinical data of epilepsy patients

\begin{tabular}{lc}
\hline Age $( \pm \mathbf{S D}) *$ & $29.2( \pm 10.9)$ \\
\hline Sex $\mathbf{n}(\%)$ & $20(66.7)$ \\
Female & $10(33.3)$ \\
Male & $18(60)$ \\
\hline Drug use $\mathbf{n}(\%)$ & $12(40)$ \\
Monotherapy & $1-51(10.5)$ \\
Polytherapy & $1-48(14.7)$ \\
\hline Disease duration (year), min-max $(\mathbf{M}) * *$ & \\
\hline Annual number of attacks, $\min -\mathbf{m a x}(\mathbf{M}) * *$ & $20(66.7)$ \\
\hline Seizure type $\mathbf{n}(\%)$ & $10(33.3)$ \\
Primary generalized seizure & \\
Secondary generalized seizure & \\
\hline$*$ SD: Standard Deviation, $* * \mathbf{M}:$ median, min: minimum, max: & \\
maximum &
\end{tabular}

Table 2. The $\alpha$-syn, MDA and MPO levels in patient and control groups

\begin{tabular}{lccc}
\hline & $\begin{array}{c}\text { Epilepsy } \\
(\mathbf{n}=\mathbf{3 0})\end{array}$ & $\begin{array}{c}\text { Control } \\
(\mathbf{n}=\mathbf{3 0})\end{array}$ & $\mathbf{p}$ \\
\hline Alpha-synuclein & 37.49 & 34.60 & 0.52 \\
$(\mathbf{n g} / \mathbf{m l}) *$ & $( \pm 11.4)$ & $( \pm 21.9)$ & \\
Malondialdehyde & 58.37 & 78.46 & $<0.001 * *$ \\
$(\mathbf{n m o l} / \mathbf{m l}) *$ & $( \pm 15.5)$ & $( \pm 24.1)$ & \\
Myeloperoxidase & 4706.41 & 6880.76 & $0.003 * *$ \\
$(\mathbf{u} / \mathbf{l}) *$ & $( \pm 2408.7)$ & $( \pm 3061.8)$ & \\
\hline *mean \pm standard deviation & &
\end{tabular}

Table 3. The $\alpha$-syn, MDA, MPO levels in monotherapy and polytherapy patients

\begin{tabular}{lccc}
\hline & $\begin{array}{c}\text { Monotherapy } \\
(\mathbf{n = 1 8})\end{array}$ & $\begin{array}{c}\text { Polytherapy } \\
(\mathbf{n = 1 2})\end{array}$ & $\mathbf{p}$ \\
\hline $\begin{array}{l}\text { Alpha-synuclein } \\
(\mathbf{n g} / \mathbf{m l}) *\end{array}$ & $36.79( \pm 11.08)$ & $38.53( \pm 12.43)$ & 0.69 \\
$\begin{array}{l}\text { Malondialdehyde } \\
(\mathbf{n m o l} / \mathbf{m l}) *\end{array}$ & $55.31( \pm 14.47)$ & $62.96( \pm 16.68)$ & 0.19 \\
$\begin{array}{l}\text { Myeloperoxidase } \\
(\mathbf{u} / \mathbf{l}) *\end{array}$ & $\begin{array}{c}4722.22 \\
( \pm 2446.73)\end{array}$ & $\begin{array}{c}4682.69( \pm 245 \\
8.26)\end{array}$ & 0.96 \\
\hline
\end{tabular}

*mean+standard deviation

Table 4. The $\alpha$-syn, MDA, MPO levels in patients with primary and secondary generalized seizures

\begin{tabular}{lccc}
\hline & $\begin{array}{c}\text { Primer } \\
\text { Generalized } \\
(\mathbf{n}=\mathbf{2 0})\end{array}$ & $\begin{array}{c}\text { Sekonder } \\
\text { Generalized } \\
(\mathbf{n}=\mathbf{1 0})\end{array}$ & $\mathbf{p}$ \\
\hline $\begin{array}{l}\text { Alpha-synuclein } \\
(\mathbf{n g} / \mathbf{m l}) *\end{array}$ & $34.9( \pm 7.6)$ & $42.6( \pm 15.9)$ & 0.08 \\
\hline $\begin{array}{l}\text { Malondialdehyde } \\
(\mathbf{n m o l} / \mathbf{m l}) *\end{array}$ & $57.3( \pm 14.3)$ & $60.4( \pm 18.4)$ & 0.61 \\
\hline $\begin{array}{l}\text { Myeloperoxidase } \\
(\mathbf{u} / \mathbf{l}) *\end{array}$ & $\begin{array}{c}4807.6 \\
( \pm 2488.7)\end{array}$ & $\begin{array}{c}4503.8 \\
( \pm 2355.9)\end{array}$ & 0.75 \\
\hline *mean+standard deviation & & \\
\hline
\end{tabular}

*mean \pm standard deviation 


\section{Discussion}

In our study, similar and different results were obtained with the literature. When the epileptic patients and healthy controls were compared, the levels of MPO and MDA were higher in the control group, which was statistically significant. The level of $\alpha$-syn was higher in the epilepsy group than in the control group but there was no statistically significant difference. There was a positive correlation between the $\alpha$-syn level and disease duration and as the duration of disease increased, the level of $\alpha$-syn also increased. There was no statistically significant correlation between the gender, seizure type, single or multiple drug use and MPO, MDA, and $\alpha$-syn levels.

MPO leads to the production of hypochlorous acid, a strong oxidant. Hypochlorous acid is a highly reactive neurotoxic oxidant. The mammalian brain is sensitive to oxidative stress. Any damage to the brain or blood-brain barrier may initiate the formation of free oxygen radicals. ${ }^{17}$ Disruption of the blood-brain barrier is effective in seizure induction and progression.18 In our study, the MPO level was lower in the epilepsy patients than in the control group. There was no statistically significant correlation between the disease duration and attack frequency and MPO level. In epileptic seizures, free radicals are overproduced, which contribute to neuronal hyperexcitability and neuronal cell death. MPO is thought to play an important role in the pathogenesis of epilepsy along with many neurodegenerative diseases. Recent experimental and human-induced resistant epilepsy studies have shown an increased prevalence of MPO in inflammatory cells, which triggers chlorinative stress in epileptogenesis. ${ }^{19}$ While MPO was expected to be high in epilepsy patients, the reason for low MPO levels in the patients in our study was thought to be due to the antiepileptic drugs used. This suggests that antiepileptics have MPO-inhibiting properties. In the studies conducted, it has been found that 4-Aminobenzoic acid hydrazide, an irreversible MPO inhibitor, provides neuroprotection in experimental epilepsy. ${ }^{19}$ This shows that there is a need for studies with larger patient populations. Comparison of MPO values at the time of diagnosis and after treatment with antiepileptic drugs will contribute to the evaluation of the efficacy of antiepileptic drugs on MPO.

Oxidative stress is a major factor in the pathogenesis of acute and chronic central nervous system damage and neuronal damage. ${ }^{20}$ Numerous studies suggest that serum electrolytes, trace elements, and lipid peroxidation play a causal role in some types of epilepsy and seizure recurrence due to oxidative stress. $^{21}$ While the MDA, a lipid peroxidation product and oxidative stress marker was expected to be high in epilepsy patients, it was higher in the control group in our study and this was statistically significant. It was thought that lower the MDA levels in epilepsy patients may be due to the antiepileptic drugs used. In this case, the MDA level should be lower in patients having polytherapy, while MDA level was higher in the patients having polytherapy than those having monotherapy. This suggests that the type of antiepileptic drug used is also important at the MDA level. In a different experimental study, it was reported that the level of MDA decreased with ethosuximide, phenytoin, and primidone. ${ }^{22}$ Studies are showing that the chronic use of antiepileptics such as valproic acid, phenytoin, and carbamazepine may increase the free radical formation and subsequently oxidative damage in neurons. ${ }^{23}$ When the drugs used by the patients in our study were grouped, no comparison could be made between the drug groups since the number of groups was small. Our patients had single and combined drug use in the form of valproic acid, carbamazepine, lamotrigine, levetirasetam, topiramate, phenytoin, lacosamide. Although many studies reported that high MDA level was associated with epilepsy, our study results did not support this. ${ }^{9,21}$ In another study, the MDA levels were found to be higher in patients treated with antiepileptic drugs compared to untreated patients and it was concluded that the drugs had oxidative stress-enhancing effects. ${ }^{24}$ In our study, all patients were using antiepileptic drugs, and the duration of drug use were different from each other and there was no statistically significant correlation between duration of drug use and the MDA levels. This suggests that the reason for the different results of our study from the literature may be due to the patients' use of different groups of drugs.

The low levels of inflammatory and oxidant markers in our patient group supported that there was no single cause in epileptogenesis, suggesting that other than oxidative and inflammatory pathways, there may be different neurogenetic pathologic pathways in resistant epilepsies. Moreover, the studies reporting that oxidative and inflammatory mechanisms are effective had been conducted with serum samples studied after the patient group had an active seizure. In this study, patients who had ongoing seizures but did not have a history of seizures in the last 24 hours were included in the study. Therefore, our results support that different pathways are effective in epileptogenesis and suggest that antiepileptic drugs used may also have anti-oxidant and anti-inflammatory effects.

$\alpha$-syn is an abundantly found neuronal protein. Although its biological function is not exactly known, the results obtained in cellular models show that they have important roles in vesicular transport and synaptic physiology. ${ }^{25} \alpha$-syn has been found abundantly in the presynaptic nerve terminals in the dentate supragranular region after the formation of pilocarpine-induced recurrent seizures.26 There is no biochemical prognostic marker used in the diagnosis and follow-up of epilepsy. The diagnosis is supported by clinical and electrophysiological tests. In our study, the $\alpha$-syn level was higher in the epileptic patients and this was not statistically significant. However, there was a positive correlation between $\alpha$-syn level and disease duration, and as the duration of disease increased, the level of $\alpha$-syn also increased $(\mathrm{r}=0.379, \mathrm{p}=0.03)$. There was no statistically significant correlation between seizure frequency and $\alpha$-syn level. In a study, the levels of $\alpha$-syn were found to be higher in the serum and CSF of patients with resistant epilepsy compared to newly diagnosed epilepsy patients, non-resistant epilepsy patients, and the control group. It has been stated that the level of $\alpha$-syn can be used as a biochemical marker for resistant epilepsy patients. ${ }^{27}$ The fact that $\alpha$-syn increased correlatedly with disease duration but did not change with seizure frequency in our study suggests that it may be more indicative of neurodegeneration but its use as a biomarker for resistant epilepsy is not reliable. Patients may have protein leak due to the deterioration of blood-brain barrier during seizure 
and the $\alpha$-syn in protein structure may also increase in serum after seizures. In our study, higher $\alpha$-syn level in the epileptic patients than in the control group and increased $\alpha$ syn level with increased duration of the disease can be explained by the deterioration of blood-brain barrier during a seizure, while the absence of correlation between the $\alpha$ syn level and seizure frequency is contrary to this. And this suggests that the doses of antiepileptic drugs used and the duration of drug use also affected the $\alpha$-syn level. Mehrabi et al. showed that metformin has anticonvulsant and antiepileptic effects in their experimental epilepsy model.28 Regarding the effect of metformin, Hussein et al. found that metformin caused downregulation of the $\alpha$-syn in hippocampal neurons and they thought that its antiepileptic effect is caused by this. ${ }^{29}$ The study by Hussein et al. showed that the $\alpha$-syn level was affected by drugs.

Study limitations; We had limitations such as the small number of patients, the use of different groups of drugs, the samples being studied in serum but not in the cerebrospinal fluid.

\section{Conclusion}

Epileptic seizures lead to an increase in oxidative stress, increasing the MPO and MDA levels. In our study, the higher MPO and MDA levels in the control group supported that antiepileptic drugs suppress oxidative stress. The absence of correlation between the seizure frequency and $\alpha$-syn level, while the $\alpha$-syn level increased with disease duration in epilepsy patients, suggests that the type, duration, and doses of drugs used affected the level of $\alpha$ syn. The fact that the $\alpha$-syn level was higher in the epilepsy patients but this was not statistically significant and was affected by drugs indicates that it is not a useful parameter as a biomarker. Further studies on biomarkers that can be used for follow-up in resistant epilepsies and that are not affected by drugs should be conducted.

\section{Acknowledgement}

None.

\section{Conflict of Interest}

We declare that we have no conflict of interest.

\section{References}

1. Reddy DS. Neuroendocrine aspects of catamenial epilepsy. Hormones and behavior; 2013. 63(2):25466. DOI: 10.1016/j.yhbeh.2012.04.016

2. Bar-Klein G, Lublinsky S, Kamintsky L, Noyman I, Veksler R, Dalipaj $\mathrm{H}$, et al. Imaging blood-brain barrier dysfunction as a biomarker for epileptogenesis. Brain; 2017. 140(6):1692-705.

DOI: 10.1093/brain/awx073

3. Aroniadou-Anderjaska V, Fritsch B, Qashu F, Braga MF. Pathology and pathophysiology of the amygdala in epileptogenesis and epilepsy. Epilepsy research; 2008. 78(2-3):102-16.

DOI: 10.1016/j.eplepsyres.2007.11.011

4. Löscher W, Brandt C. Prevention or modification of epileptogenesis after brain insults: experimental approaches and translational research. Pharmacological reviews; 2010. 62(4):668-700.

DOI: $10.1124 /$ pr. 110.003046

5. Ashrafi MR, Shams S, Nouri M, Mohseni M, Shabanian R, Yekaninejad MS, et al. A probable causative factor for an old problem: selenium and glutathione peroxidase appear to play important roles in epilepsy pathogenesis. Epilepsia; 2007. 48(9):17505. DOI: $10.1111 / \mathrm{j} .1528-1167.2007 .01143 . x$

6. Vezzani A, French J, Bartfai T, Baram TZ. The role of inflammation in epilepsy. Nature reviews neurology; 2011. 7(1):31. DOI:10.1038/nrneurol.2010.178

7. Fabene PF, Mora GN, Martinello M, Rossi B, Merigo F, Ottoboni L, et al. A role for leukocyte-endothelial adhesion mechanisms in epilepsy. Nature medicine; 2008. 14(12):1377-83. DOI: 10.1038/nm.1878. Epub

8. Van der Veen BS, de Winther MP, Heeringa P. Myeloperoxidase: molecular mechanisms of action and their relevance to human health and disease. Antioxidants \& redox signaling; 2009. 11(11):2899937. DOI: 10.1089/ars.2009.2538

9. Pandey MK, Mittra P, Maheshwari P. The lipid peroxidation product as a marker of oxidative stress in epilepsy. J Clin Diagn Res; 2012. 6(4):590-92. Avalaible from: https://www.jcdr.net/articles/pdf/2134/11\%20\%204228.pdf

10. Nisha Y, Bobby Z, Wadwekar V. Biochemical derangements related to metabolic syndrome in epileptic patients on treatment with valproic acid. Seizure; 2018. 60:57-60.

DOI: 10.1016/j.seizure.2018.06.003

11. Shichiri M. The role of lipid peroxidation in neurological disorders. Journal of clinical biochemistry and nutrition; 2014. 14-0.

DOI: $10.3164 /$ jcbn. $14-10$

12. Andreu-Cervera A, Anselme I, Karam A, Laclef C, Catala M, Schneider-Maunoury S. The ciliopathy gene Ftm/Rpgrip1l controls mouse forebrain patterning via region-specific modulation of Hedgehog/Gli signaling. Journal of Neuroscience; 2019. 39(13):2398-415. DOI: 10.1523/JNEUROSCI.219918.2019

13. Almandoz-Gil L, Welander H, Ihse E, Khoonsari PE, Musunuri S, Lendel C, et al. Low molar excess of 4oxo-2-nonenal and 4-hydroxy-2-nonenal promote oligomerization of alpha-synuclein through different pathways. Free Radical Biology and Medicine; 2017. 110:421-31.

DOI: 10.1016/j.freeradbiomed.2017.07.004

14. Liu G, Zhang C, Yin J, Li X, Cheng F, Li Y, et al. $\alpha-$ Synuclein is differentially expressed in mitochondria from different rat brain regions and dose-dependently down-regulates complex I activity. Neuroscience letters; 2009. 454(3):187-92.

DOI: 10.1016/j.neulet.2009.02.056

15. Bradley P, Da P. Christensen RD, Rothstein G: Measurement of cutaneous inflammation: Estimation of neutrophil content with an enzyme marker. J invest Dermatol; 1982. 78:206-9. DOI: 10.1111/15231747.ep12506462. 
16. Ohkawa $\mathrm{H}$, Ohishi $\mathrm{N}$, Yagi K. Assay for lipid peroxides in animal tissues by thiobarbituric acid reaction. Analytical biochemistry; 1979. 95(2):351-8. DOI: 10.1016/0003-2697(79)90738-3

17. Sorce S, Krause K-H. NOX enzymes in the central nervous system: from signaling to disease. Antioxidants \& redox signaling; 2009. 11(10):2481504. DOI: 10.1089 /ars.2009.2578

18. Van Vliet E, Araujo S, Redeker S, van Schaik R, Aronica E, Gorter J. Long-lasting increased permeability of the blood-brain barrier may contribute to seizure progression in temporal lobe epilepsy: 4.110. Epilepsia; 2006. 47.

DOI: $10.1093 /$ brain/awl318

19. Zhang Y, Seeburg DP, Pulli B, Wojtkiewicz GR, Bure $\mathrm{L}$, Atkinson $\mathrm{W}$, et al. Myeloperoxidase nuclear imaging for epileptogenesis. Radiology; 2016. 278(3):822-30. DOI: 10.1148/radiol.2015141922

20. Uttara B, Singh AV, Zamboni P, Mahajan R. Oxidative stress and neurodegenerative diseases: a review of upstream and downstream antioxidant therapeutic options. Current neuropharmacology; 2009. 7(1):65-74.

DOI: $10.2174 / 157015909787602823$

21. Guler SK, Aytac B, Durak ZE, Cokal BG, Gunes N, Durak I, et al. Antioxidative-oxidative balance in epilepsy patients on antiepileptic therapy: A prospective case-control study. Neurological Sciences; 2016. 37(5):763-7. DOI: 10.1007/s10072016-2494-0

22. Bogdanov G, Mishchenko D, Kotel'nikova R, Frog E, Faĭngol'd I, Tat'ianenko L, et al. Anticonvulsants as bioantioxidants under stress conditions. Biomeditsinskaia Khimiia; 2009. 55(4):519-24. Avalaible from:
23. Martinc B, Grabnar I, Vovk T. The role of reactive species in epileptogenesis and influence of antiepileptic drug therapy on oxidative stress. Current Neuropharmacology; 2012. 10(4):328-43. DOI: $10.2174 / 157015912804499447$

24. Prasad D, Satyanarayana U, Shaheen U, Prabha TS, Munshi A. Oxidative stress in the development of genetic generalised epilepsy: an observational study in southern Indian population. Journal of Clinical and Diagnostic Research: JCDR; 2017. 11(9):BC05.

DOI: $10.7860 / J C D R / 2017 / 29133.10604$

25. Greten-Harrison B, Polydoro M, Morimoto-Tomita M, Diao L, Williams AM, Nie EH, et al. $\alpha \beta \gamma$-Synuclein triple knockout mice reveal age-dependent neuronal dysfunction. Proceedings of the National Academy of Sciences; 2010. 107(45):19573-8.

DOI: $10.1073 /$ pnas.1005005107

26. Li A, Choi YS, Dziema H, Cao R, Cho HY, Jung YJ, et al. Proteomic profiling of the epileptic dentate gyrus. Brain pathology; 2010. 20(6):1077-89.

DOI: $10.1111 / \mathrm{j} .1750-3639.2010 .00414 . \mathrm{x}$

27. Rong $\mathrm{H}$, Jin L, Wei W, Wang X, Xi Z. Alphasynuclein is a potential biomarker in the serum and CSF of patients with intractable epilepsy. Seizure; 2015. 27:6-9. DOI: 10.1016/j.seizure.2015.02.007

28. Mehrabi S, Sanadgol N, Barati M, Shahbazi A, Vahabzadeh G, Barzroudi M, et al. Evaluation of metformin effects in the chronic phase of spontaneous seizures in pilocarpine model of temporal lobe epilepsy. Metabolic Brain Disease; 2018. 33(1):10714. DOI:10.1007/s11011-017-0132-z

29. Hussein AM, Eldosoky M, El-Shafey M, El-Mesery M, Ali AN, Abbas KM, et al. Effects of metformin on apoptosis and $\alpha$-synuclein in a rat model of pentylenetetrazole-induced epilepsy. Canadian Journal of Physiology and Pharmacology; 2019. 97(1):37-46. DOI: $10.1139 /$ cjpp-2018-0266 